\title{
Morphological Study of Insect Mechanoreceptors to Develop Artificial Bio-Inspired Mechanosensors ${ }^{\dagger}$
}

\author{
Shashikanth Chakilam ${ }^{1, *}$, Dan Ting Li ${ }^{2}$, Zhang Chuan Xi ${ }^{2}$, Rimvydas Gaidys ${ }^{1}$ \\ and Audronè Lupeikiené ${ }^{3}$ \\ 1 Faculty of Mechanical Engineering, Kaunas University of Technology, Kaunas 44249, Lithuania; \\ e-mail1@e-mail.com \\ 2 Institute of Insect Science, Zhejiang University, Hangzhou 310058, China; e-mail1@e-mail.com (D.T.L.); \\ e-mail2@e-mail.com (Z.C.X.) \\ 3 Institute of Mathematics and Informatics, Vilnius University, Vilnius 01513, Lithuania; e-mail1@e-mail.com \\ * Correspondence: shashikanth.chakilam@ktu.edu; Tel.: +370-63626699 \\ + Presented at the 7th International Electronic Conference on Sensors and Applications, 15-30 November \\ 2020; Available online: https://ecsa-7.sciforum.net/.
}

Published: 15 November 2020

\begin{abstract}
Mechanoreceptors of the insect play a vital role in insect to sense and monitor the environmental parameters like flow, tactile pressure, etc. This paper presents the studies made on the morphology of the mechanoreceptor of insect Blattella Asahinai (scientific name of cockroach), which is a hair-like structure known as trichoid sensilla, by Scanning Electron Microscope and Confocal Laser Microscope. The scanned images show the details of sensilla components in which the hair is embedded in the socket which are connected with the cuticle and joint membrane where the dendrite touches at the base of the hair passing through the cuticle layers. The images also show that the tubular bodies and microtubules are tightly compacted inside the dendrite. This paper presents the details of how the sensilla works when an external stimulus act on it. The hair deflects with the disturbance of the cuticle and joint membrane and this deformed hair lean on the dendrite which is attached at the base of the hair which in turn presses the tubular bodies and microtubules which develop negative ions passing down through the dendrite to the neuron which provides information as an electric signal to the brain of the insect so that it responds for necessary action. Based on the morphological studies, sensing mechanism, material properties of the components, design principles will be evolved for the development of an artificial bio-inspired sensor. A solid works model of the sensilla is also presented.
\end{abstract}

Keywords: mechanosensors; sensilla; Bio inspired sensors; confocal microscope; scanning electron microscope

\section{Introduction}

The insects are ultrasensitive in sensing the environmental changes mainly by mechanical stimuli like air flow, vibration, pressure changes, etc., by using their specialized sensory organs which are known as mechanoreceptors. These are hair like structures technically called as sensilla. These mechanoreceptors spread all over the body and mostly on the exoskeleton surfaces which are projected from the cuticle. Forces due to external disturbances around the hair of the insect will develop stresses inside the walls of the cuticle. These mechanoreceptors present on the cuticle surface will have different sizes and shapes and sensitivity based on their location on the body of the insect. Trichoid sensilla are one amongst them which exist on all body parts of the insect. They help in detecting the forces developed internally and externally when the hair deflects [1]. Antennae are the foremost sensory parts in the insect on which the trichoid sensilla are abundantly found. These 
sensilla have a simple external and complex internal construction in contrast to the chemosensillar. The external part of the trichoid sensilla senses the changes in air flow and contact with other surfaces. Usually the hairs are flexible though some are stiff. When the hair bends, it presses against the joint membrane, the dendrite and the tubular bodies and sends signal to the nerve cells [2].

The external and internal morphological studies of these mechanoreceptors are performed by different electron microscopes. Due to their high resolution and magnification, the studies made through scanning electron microscope (SEM), transmission electron microscope (TEM) on the mechanoreceptors of the insects by many researchers have contributed for the vast knowledge on their external and internal morphology of sensilla [3].

The present studies on the morphology of sensilla and its sensing behaviour will help in replicating the features for developing the sensors using MEMS technology. Several researchers have worked on the artificial hair [4-6] through bio-inspired insect mechanoreceptors by modelling them as an inverted pendulum. Some have developed biomimetic flow sensitive sensors, biomimetic accelerometers, etc. These bio-inspired mechanoreceptors have been fabricated using surface machining, 3D printing, photolithography and MEMS technologies [7-9].

The external and internal structure and details of functioning of the trichoid sensilla of the Blattella Asahinai insect are studied during an external stimulus by SEM, TEM and are presented in this paper.

\section{Materials and Methods}

\subsection{Insects}

The Blattella Asahinai insects are collected from the bank of Musi River in Hyderabad, India and are preserved in the laboratory of Biological Regional Centre of Zoological Survey of India, Hyderabad, India. These insects are reared at a temperature of $22{ }^{\circ} \mathrm{C}$ till they develop their cerci and full length of antennas. The insects of identical growth are selected for the studies.

\subsection{Preparation of Samples}

The selected cockroach is anesthetized by keeping it on an ice block for about a minute. The antennal segments are separated from the surface of the body using scissors and tweezers and are kept in $1 \mathrm{~N}$ potassium hydroxide solution prepared by dissolving $10 \mathrm{mg}$ of $\mathrm{KOH}$ pellets in $\mathrm{H}_{2} \mathrm{O}$. This $\mathrm{KOH}$ solution dissolves the unwanted soft tissue leaving behind the hard cuticle which is then washed with $60 \%, 70 \%$ ethanol at room temperature for about $5 \mathrm{~min}$. The antennal tissues are laterally dissected and transferred into $\mathrm{C}_{8} \mathrm{H}_{8} \mathrm{O}_{3}$ methyl salicylate solution to make the sample more transparent to get clear images under the microscope. These samples are dried well by placing them over filter paper.

\subsection{Samples under SEM, TEM}

Dried antennae tissues are placed over the surface of aluminium stubs and sticking them with a carbon tape. A slight gold coating is done on to the tissue with a sputter coater (LEICA EM ACE 200). The morphology of the sensilla on each antenna is examined under FE-SEM (Quanta FEG 250, LEICA EM TP) at an electron beam voltage of $5 \mathrm{KV}$. To observe the internal morphology, the mechanosensilla is removed from the antennae of the insect and embedded on to the Epon blocks and then placed under Ultramicrotome (LEICA EM UC7) to make the ultrathin sections. Ultrathin sections of the samples are observed under TEM (FEI Technai TF20) with magnification of $25 \times$ to 2,000,000×. The images captured from both SEM, TEM are edited by using Adobe Photoshop. 


\section{Results}

\subsection{Sensilla on Antennal Segments External MORPHOLOGY}

The sensilla on the antennal segments are more in number on the distal end of the segment when compared with other segments. There are several types of sensillas present on the surface of the antennal segment, amongst those the trichoid sensilla's are large in number. Sensilla of different types are distributed over the surface shown in Figure 1.Trichoid sensilla are one of the types of sensillas have different shapes. Based on their length and shape, they are classified into two groups such as S.t. I, S.t. II. The trichoid sensillas-S.t.I have pointed apex. Trichoid sensillas-s.t.II are another type which are abundant on the segmental surface. They have curved shape with blunt tips as shown in Figure 1-I. Sensilla Basiconica (s.b) are s.b I type of sensillas with blunt tips and stout pegs. The s.b II type sensillas are very short with a bulgy base. The s.b III type sensillas are longer with sharp tips which rise from side walls of antennal surface as shown in Figure 1-II. In Figure 1-III, another type of sensilla with a blunt tip and stout pegs called sensilla chaetica (s.c) are present over the length of the antennae.

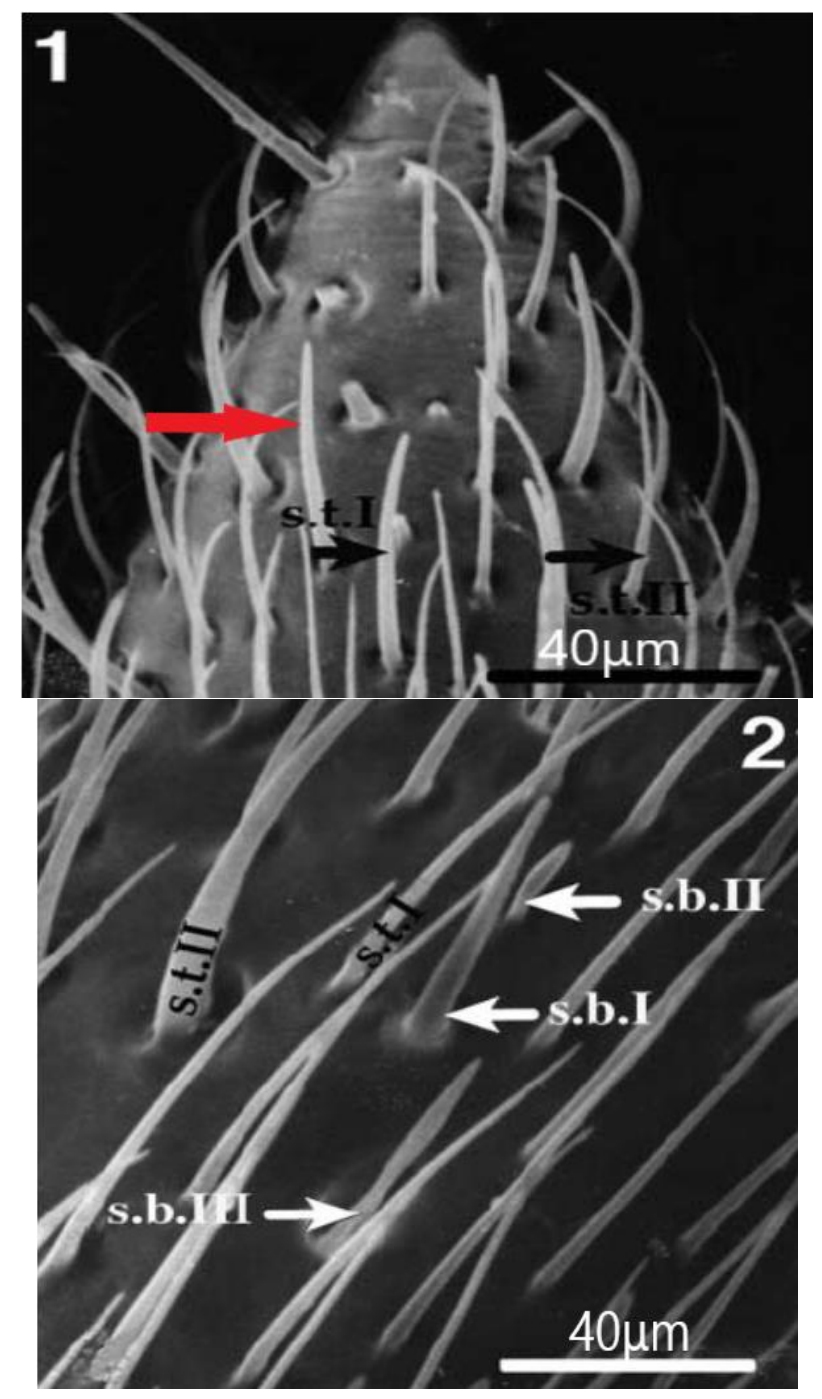




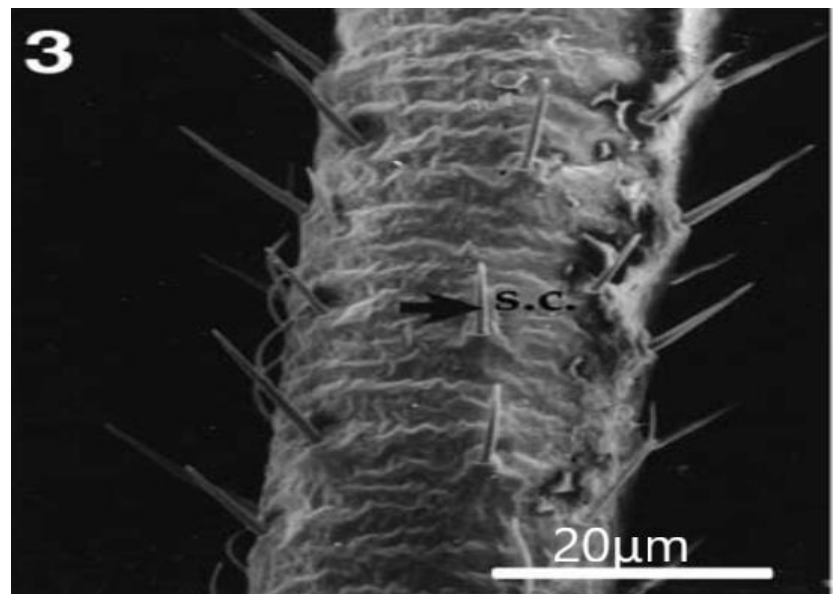

Figure 1. SEM images of External morphology of sensilla on antenna and its types. (1) Conical projection of the insect Blattella Asahinai Antennal segment. s.t: Sensilla trichoid. Indicated by short arrow s.t.I is type of trichoid with pointed apex, long arrow s.t.II is trichoid sensilla which curved and have blunt tips. (2) Apical antenna segment shows the S.Trichoid I,II and also another type of sensilla which are distributed on the surface i.e s.b Sensilla. Basiconica s.b.I, s.b.II and s.b.III. (3) Black arrow shows another type of sensilla i.e s.c Sensilla chaetica which is distributed along the axis of the antennal segment with some angle.

\subsection{Internal Morphology of the Sensilla}

The red arrow in Figure 1-I indicates the sensulla trochoid for which the internal morphology is indicated in Figure 2. The hollow hair is seen to be rising from the cuticle with the base of the hair being supported with the joint membrane and socket septum. They are connected with few suspension fibres which restrict the extent of hair base movement $[10,11]$.
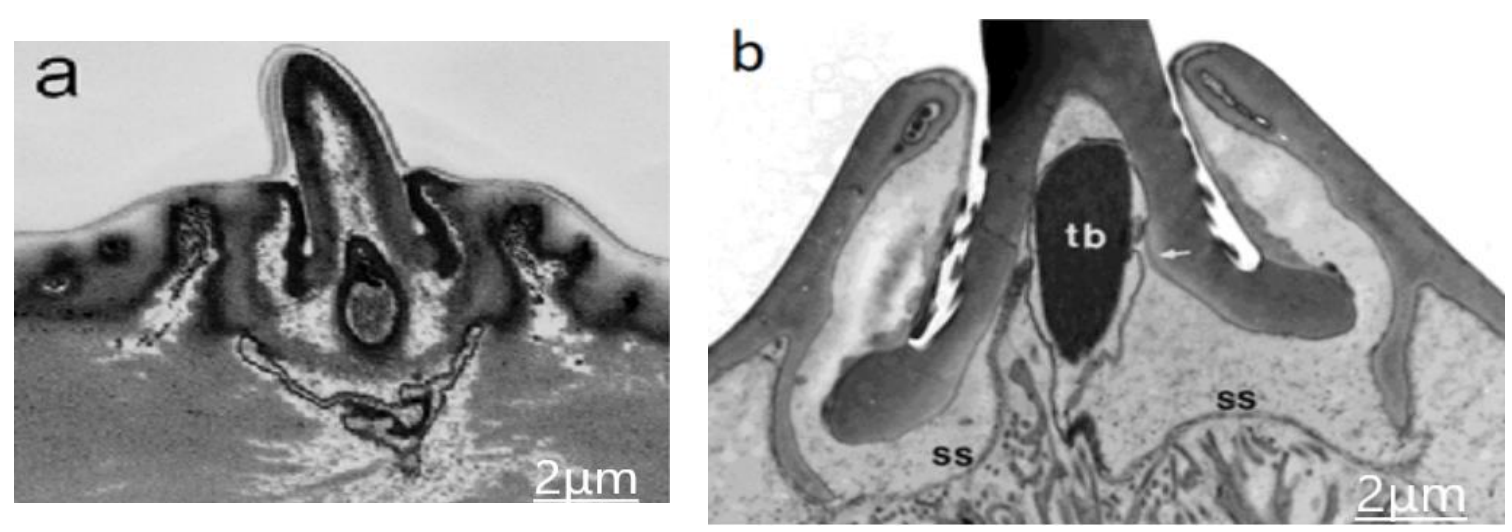

Figure 2. TEM images of internal morphology of the Trichoid sensilla (a) The cross-sectional view of the sensillum (b) Sectional view of the tubular body, socket septum. Scale line: $2 \mu \mathrm{m}$. 


\subsection{Proposed Artificial Sensilla Model}

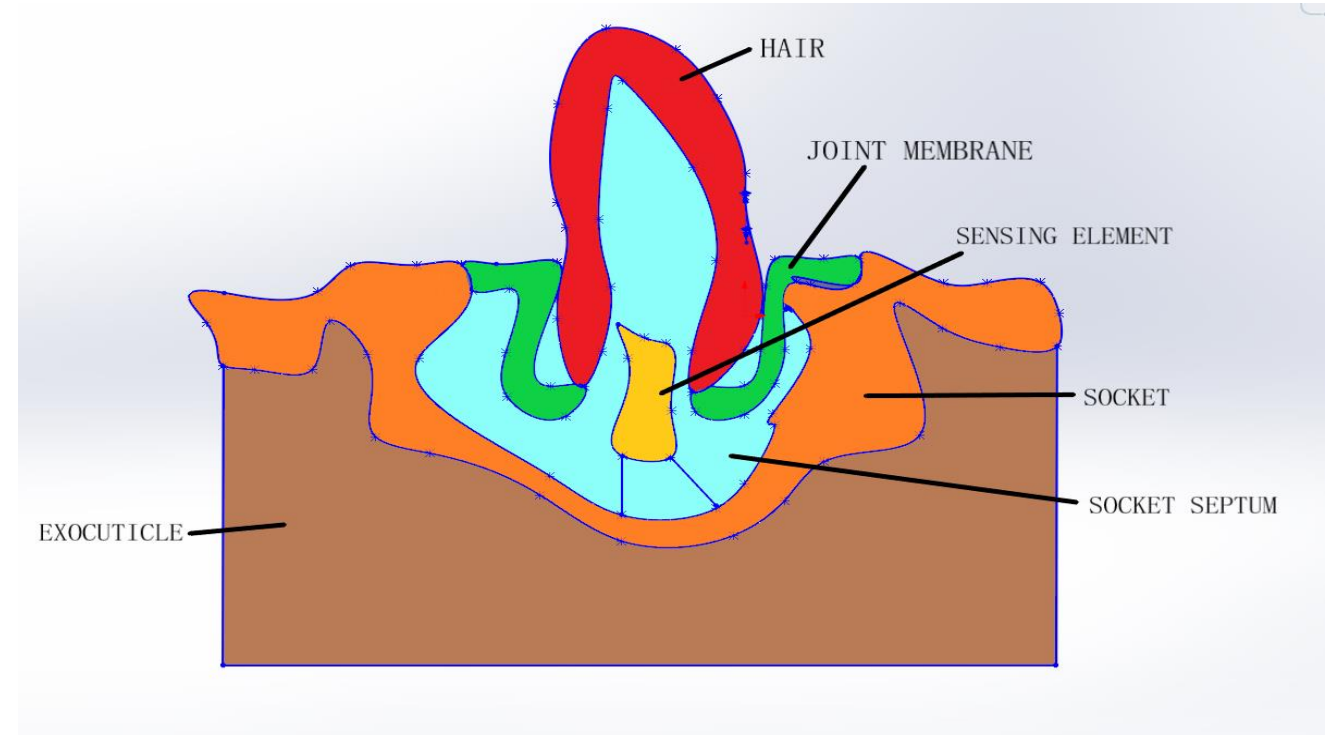

Figure 3. The Cross section of Sensilla model.

\section{Discussion}

In Blattella Asahinai, sensilla on Antenna's are of various shapes and sizes and are varies from one insect to another but the internal morphology of all types of sensilla's are same for all insects [12].

\subsection{Development of the Sensilla}

The development of sensilla starts with the division of cells which are attached bellow the cuticle in to sensory mother cell (SMC) and trichogen and tormogen mother cell (TMC). SMC further divides into sensory neuron (SC) and thecogen (Th), whereas TMC divides into trichogen cell (Tr) and tormogen cells (To). The dendrite and neuron are developed from thecogen cell (Th) and sensory neuron (Sc). The other parts of the sensilla like the hair base, joint membrane, socket, socket septum are developed by the TMC cells mutation [13-15].

In Figure 2, the socket appears on the cuticle surface from which the hollow hair rises. The joint membrane is a hollow elastic cylinder with one end connected to the base of the hair and the other end is connected to the socket. The base of the hair is supported by the joint membrane and socket septum along with few suspension fibres, which are helpful in hair movements due to their elastic nature. The distal end of the dendrite is slightly in touch with the hair base where the dendrite is covered with a dendrite sheath [16]. Main sensory part of the sensilla is the dendrite which is circular in cross-section. The tubular bodies are embedded in the dendrite which are tightly packed with the electron-dense material known as microtubules.

\subsection{Functioning of Sensilla}

Whenever an external disturbance occurs in the air, that flows over the hair, then the hair deflects slightly. As the dendrite is in touch at the base of the hollow hair, this deflection of hair will press the dendrite as well as tubular body and microtubules results in electrical charging and downward flow of ions in the microtubules from the tubular body to the neurons sending the information to the brain of the insect for taking necessary action $[12,16,17]$.

\subsection{Design of Artificial Mechanosensors}

The dimensions of the sensilla were measured using the SEM and TEM and found that the long axis of the socket is about $5 \pm 0.5 \mu \mathrm{m}$, the short axis of the socket is about $4 \pm 0.5 \mu \mathrm{m}$, the length of the hair is $15 \pm 2 \mu \mathrm{m}$, diameter is $900 \pm 150 \mathrm{~nm}$, the dendrite cross-section is round, and its diameter is about $600 \mathrm{~nm}$, the tubular body inside this dendrite is about the length of $850 \mathrm{~nm}$ and the diameter is 
of $500 \mathrm{~nm}$ and microtubules are of $50 \mathrm{~nm}$ each in diameter which is tightly packed in the tubular body. By considering the morphology, functioning, and dimensions of the sensilla, the artificial mechanosensor is designed as shown in Figure 3. The material of the hair is keratin which is a protein nanofiber containing sulfur. The mechanical properties of the hair are Density of $1100 \mathrm{~kg} / \mathrm{m}^{3}$, Low elastic modulus is $6 \mathrm{Gpa}$, High elastic modulus is $9 \mathrm{Gpa}$, Poisons ratio is 0.38 . Joint membrane and socket are the viscoelastic materials that have the young's modulus of $50 \mathrm{Mpa}$, Poisson's ratio is of 0.4. Suspension fibers are fibrous materials which are elastic in nature [18]. The dendrite is covered with dendrite sheath, and tubular body containing microtubules are the sensing elements which are of piezoelectric material.

\section{Conclusions}

In this paper, the morphology and physiology of sensilla of Blattella Asahinai the and its functioning are explained when an external stimulus act on it. The model of the bio-inspired artificial mechanosensor is presented. The mathematical model and simulation studies on designed artificial hair sensor for flow and tactile parameters will be made in the future.

\section{References}

1. George, A.; Nagy, B.A.L. Morphology, distribution, and ultrastructural differences of sensilla trichodea and basiconica on the antennae of the oriental fruit moth, Grapholitha molesta (Busck) (Lepidoptera: Tortricidae). Int. J. Insect Morphol. Embryol. 1984, 13, 157-170. doi:10.1016/0020-7322(84)90023-0.

2. Shimozawa, T.; Kanou, M. The aerodynamics and sensory physiology of range fractionation in the cereal filiform sensilla of the cricket Gryllus bimaculatus. J. Comp. Physiol. 1984, 155, 495-505. doi:10.1007/BF00611914.

3. Dey, S.; Hooroo, R.N.; Wankhar, D. Scanning electron microscopic studies of the external morphology of sensilla on the legs of a butterfly, Graphium sarpedon (Lepidoptera-Papillionidae). Micron 1995, 26, 367376. doi:10.1016/0968-4328(95)00013-5.

4. Dijkstra, M.; van Baar, J.J.; Wiegerink, R.J.; Lammerink, T.S.J.; de Boer, J.H.; Krijnen, G.J.M. Artificial sensory hairs based on the flow sensitive receptor hairs of crickets. J. Micromech. Microeng. 2005, 15, S132S138.

5. Dagamseh, A.M.K. Estimation of squeeze film damping in artificial hair-sensor towards the detection-limit of crickets' hairs. Microsyst. Technol. 2014, 20, 963-970. doi:10.1007/s00542-014-2099-6.

6. Krijnen G.J.M.; Droogendijk H.; Dagamseh A.M.K.; Jaganatharaja R.K.; Casas J. Crickets as Bio-Inspiration for MEMS-Based Flow-Sensing. In Flow Sensing in Air and Water; Bleckmann, H., Mogdans, J., Coombs, S., Eds.; Springer: Berlin/Heidelberg, Germany, 2014; pp. 459-488. doi:10.1007/978-3-642-41446-6_17.

7. Krijnen, G.J.M.; Lammerink, T.; Wiegerink, R. Learning from crickets: Artificial hair-sensor array developments. Sensors 2010, 2010, 2218-2223. IEEE, Kona, HI.

8. Krijnen, G.; Lammerink, T.; Wiegerink, R.; Casas, J. Cricket Inspired Flow-Sensor Arrays. Sensors 2007, 2007, 539-546, IEEE, Atlanta, GA, doi:10.1109/ICSENS.2007.4388455.

9. Droogendijk, H.; Casas, J.; Steinmann, T.; Krijnen, G.J. Performance assessment of bio-inspired systems: Flow sensing MEMS hairs. Bioinspir. Biomim. 2014, 10, 016001.

10. Droogendijk, H. Bio-Inspired MEMS Flow and Inertial Sensors; University of Twente: Enschede, The Netherlands, 2014, doi:10.3990/1.9789036535984.

11. di Giulio, A.; Maurizi, E.; Valerio, M.; Stacconi, R.; Romani, R. Functional structure of antennal sensilla in the myrmecophilous beetle Paussus favieri (Coleoptera, Carabidae, Paussini). Micron 2012, 43, 705-719, doi:10.1016/j.micron.2011.10.013.

12. Keil, T.A. Functional morphology of insect mechanoreceptors. Microscopy Res. Tech. 1997, 39, 506-531.

13. Letourneau, P.C.; Condic, M.L.; Snow, D.M. Interactions of developing neurons with the extracellular matrix. J. Neurosci. 1994, 14, 915-928. doi:10.1523/JNEUROSCI.14-03-00915.1994.

14. Orgogozo, V.; Grueber, W.B. FlyPNS, A database of the Drosophila embryonic and larval peripheral nervous system. BMC Dev. Biol. 2005, 5, 4. doi:10.1186/1471-213X-5-4.

15. Giangrande A.; Palka J. Genes involved in the development of the peripheral nervous system of Drosophila. Seminars Cell Biol. 1990, 1, 197-209. 
16. Gnatzy, W.; Weber, K.M. Tormogen cell and receptor-lymph space in insect olfactory sensilla. Cell Tissue Res. 1978, 189, 549-554. doi:10.1007/BF00209140.

17. Fred, T.; Hartley, D.A. Inhibition of cell fate in Drosophila by Enhancer of split genes. Mech. Dev. 1995, 51, 305-315.

18. Barth, F.G. Spider mechanoreceptors. Curr. Opin. Neurobiol. 2004, 4, 415-422.

Publisher's Note: MDPI stays neutral with regard to jurisdictional claims in published maps and institutional affiliations.

(C) 2020 by the authors. Submitted for possible open access publication under the terms and conditions of the Creative Commons Attribution (CC BY) license (http://creativecommons.org/licenses/by/4.0/). 